Wright State University

CORE Scholar

$11-1-2001$

\title{
Thermal Stability of Isolated and Complexed Ga Vacancies in GaN Bulk Crystals
}

\author{
K. Saarinen \\ T. Suski \\ I. Grzegory \\ David C. Look \\ Wright State University - Main Campus, david.look@wright.edu
}

Follow this and additional works at: https://corescholar.libraries.wright.edu/physics

Part of the Physics Commons

\section{Repository Citation}

Saarinen, K., Suski, T., Grzegory, I., \& Look, D. C. (2001). Thermal Stability of Isolated and Complexed Ga Vacancies in GaN Bulk Crystals. Physical Review B, 64 (23), 233201.

https://corescholar.libraries.wright.edu/physics/181

This Article is brought to you for free and open access by the Physics at CORE Scholar. It has been accepted for inclusion in Physics Faculty Publications by an authorized administrator of CORE Scholar. For more information, please contact library-corescholar@wright.edu. 


\title{
Thermal stability of isolated and complexed Ga vacancies in GaN bulk crystals
}

\author{
K. Saarinen \\ Laboratory of Physics, Helsinki University of Technology, P.O. Box 1100, FIN-02015 HUT, Finland \\ T. Suski and I. Grzegory \\ UNIPRESS, High Pressure Research Center, Polish Academy of Sciences, 01-142 Warsaw, Poland \\ D. C. Look \\ Air Force Research Laboratory, Materials and Manufacturing Directorate, and Semiconductor Research Center, \\ Wright State University, Dayton, Ohio \\ (Received 4 September 2001; published 27 November 2001)

\begin{abstract}
We have applied positron annihilation spectroscopy to show that 2-MeV electron irradiation at $300 \mathrm{~K}$ creates primary Ga vacancies in GaN with an introduction rate of $1 \mathrm{~cm}^{-1}$. The Ga vacancies recover in long-range migration processes at 500-600 K with an estimated migration energy of 1.5 (2) eV. Since the native Ga vacancies in as-grown GaN survive up to much higher temperatures (1300-1500 K), we conclude that they are stabilized by forming complexes with oxygen impurities. The estimated binding energy of 2.2 (4) eV of such complexes is in good agreement with the results of theoretical calculations.
\end{abstract}

DOI: 10.1103/PhysRevB.64.233201

PACS number(s): 61.72.Ji, 61.82.Fk, 66.30.Lw, 78.70.Bj

Gallium nitride and its alloys form a class of important semiconductor materials for applications in optoelectronics at blue wavelength and electronic devices operating at high temperature and high voltage. The growing interest in intrinsic point defects in GaN is stimulated by their role in determining the electrical and optical properties of the material, as well as by their importance and behavior in various processing steps such as ion implantation and thermal annealing. Theoretical calculations predict that $\mathrm{Ga}$ vacancies and related complexes form the predominant class of point defects in $n$-type GaN. ${ }^{1,2}$ These defects have been experimentally observed by positron annihilation spectroscopy., $\mathrm{Ga}$ vacancies are electrically active acceptors, and involved in the optical transition leading to the emission of yellow luminescence light. ${ }^{1,3}$

Although Ga vacancies have been observed as important native defects, the experiments so far have given only limited information on their formation mechanism during the crystal growth. According to calculations, both isolated and complexed $\mathrm{Ga}$ vacancies are abundantly created in $n$-type $\mathrm{GaN}$, and the binding energies of associated vacancyimpurity complexes such as $V_{\mathrm{Ga}}-\mathrm{O}_{\mathrm{N}}$ and $V_{\mathrm{Ga}}-\mathrm{Si}_{\mathrm{Ga}}$ have been theoretically estimated. ${ }^{1,2}$ However, it is not known whether isolated or complexed vacancies are stable at growth temperature (typically $>1300 \mathrm{~K}$ ) or survive during the cooling down of the crystals. The thermal stability of defects associated with $\mathrm{Ga}$ vacancies is determined by the migration and binding energies, but no previous experimental estimates exist for these quantities in $\mathrm{GaN}$.

Electron irradiation is a very valuable technique to introduce basic point defects in a controlled way. The annealing treatments of the irradiated material yield important information on the thermal stability of isolated intrinsic point defects. Such knowledge can be compared with the properties of native defects present in as-grown material in order to understand their atomic structure and formation mechanisms during the growth. In $\mathrm{GaN}$, electrical experiments have shown that $0.7-1.0-\mathrm{MeV}$ electrons create intrinsic donors and acceptors at equal rates of about $1 \mathrm{~cm}^{-1}$. Since electrons at these energies induce displacements mainly in the $\mathrm{N}$ sublattice, the donor and acceptor defects have been attributed to $\mathrm{N}$ vacancy and interstitial, respectively. ${ }^{5}$ After irradiation with $>1-\mathrm{MeV}$ electrons defects in the Ga sublattice can also be expected. Indeed, Chow et al. ${ }^{6}$ used optical detection of electron paramagnetic resonance (ODEPR) to observe interstitial $\mathrm{Ga}$ atoms after such irradiations. At $300 \mathrm{~K}$ the $\mathrm{Ga}$ interstitials are trapped by other defects, ${ }^{6-8}$ perhaps by the oxygen atoms present as impurities in $\mathrm{GaN}^{6}{ }^{6,9}$ The creation of $\mathrm{Ga}$ interstitials should be accompanied by the formation of vacant Ga lattice sites, which have, however, escaped direct experimental observation so far.

In this Brief Report we apply positron annihilation spectroscopy to identify defects formed in the $2-\mathrm{MeV}$ electron irradiation of GaN bulk crystals. Positrons are trapped at neutral and negative vacancies because of the missing positive charge of the ion cores. The reduced electron density at vacancies increases the positron lifetime. We show that $2-\mathrm{MeV}$ electron irradiation at $300 \mathrm{~K}$ creates $\mathrm{Ga}$ vacancies with an introduction rate of $1 \mathrm{~cm}^{-1}$. The Ga vacancies recover in heat treatments at 500-600 K. Since the ODEPR experiments ${ }^{6}$ indicate that $\mathrm{Ga}$ interstitials are stable and trapped by other defects at these temperatures, we conclude that $\mathrm{Ga}$ vacancies become mobile and recover in long-range migration processes with an activation energy of $1.5(2) \mathrm{eV}$. Since native Ga vacancies formed during the crystal growth of $\mathrm{GaN}$ are stable up to much higher temperatures (1300$1500 \mathrm{~K}$ ), we conclude that they are complexes with oxygen, and estimate their binding energy to be 2.2 (4) eV.

Bulk GaN crystals were grown at a nitrogen pressure of $1.5 \mathrm{GPa}$ and a temperature of $1500{ }^{\circ} \mathrm{C} .{ }^{10}$ Semi-insulating Mg-doped samples were chosen for the experiment, since these are free of native Ga vacancies as shown earlier. ${ }^{11}$ The $\mathrm{Mg}$ and $\mathrm{O}$ concentrations of the samples were [O] $\approx[\mathrm{Mg}] \approx 10^{20} \mathrm{~cm}^{-3}$, according to secondary-ion-mass 


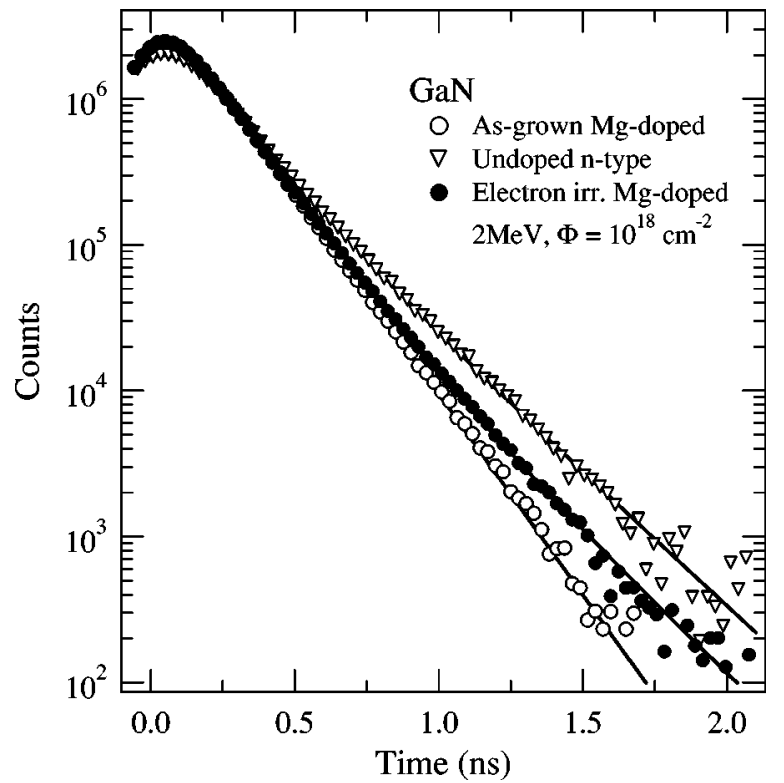

FIG. 1. Positron lifetime spectra in Mg-doped GaN before and after electron irradiation. The spectrum obtained in as-grown $n$-type GaN crystals (Ref. 11) is shown for comparison. The solid lines are fits to the sum of exponential decay components convoluted with the resolution function of the positron lifetime spectrometer.

spectrometry. ${ }^{11}$ The samples were irradiated with $2-\mathrm{MeV}$ electrons at $300 \mathrm{~K}$ to two fluences of $\Phi=3 \times 10^{17}$ and 1 $\times 10^{18} \mathrm{~cm}^{-2}$. To study the recovery of irradiation-induced defects and the temperature dependence of the positron lifetime spectrum simultaneously, we annealed the samples at temperatures $T_{\mathrm{ann}}$, and thereafter measured positron lifetimes as a function of measurement temperature $T_{\text {meas }}$ from $80 \mathrm{~K}$ up to $T_{\text {ann }}-50 \mathrm{~K}$. The 30 -min annealings were done in situ in the $10^{-3}$-mbar vacuum of the positron measurement cryostat.

The positron lifetime experiments were performed using conventional instrumentation (a time resolution of $230 \mathrm{ps}$ ) and a $30-\mu \mathrm{Ci}^{22} \mathrm{Na}$ positron source deposited onto a $1.5-\mu \mathrm{m}$ Al foil. ${ }^{12,13}$ After subtracting the constant background and positron annihilations in the source material $(210 \mathrm{ps}, 2.0 \%$; $400 \mathrm{ps}, 3.7 \%$; $2000 \mathrm{ps}, 0.15 \%)$ the lifetime spectrum $n(t)$ (examples in Fig. 1) was analyzed as the sum of exponential decay components $n(t)=\Sigma I_{i} \exp \left(-t / \tau_{i}\right)$. The positron in state $i$ (e.g., the delocalized state in the lattice or localized state at a vacancy) annihilates with a lifetime $\tau_{i}$ and an intensity $I_{i}$. A direct sign of vacancy defects in the sample is obtained when the average lifetime $\tau_{\mathrm{av}}=\Sigma I_{i} \tau_{i}$ increases above the lifetime $\tau_{B}$ obtained in the defect-free lattice.

In a positron lifetime experiment, positrons enter the sample and thermalize at $t=0$. The vertical axis of the positron lifetime spectrum (Fig. 1) shows the number of positron-electron annihilations at a time channel of $25 \mathrm{ps}$. In unirradiated $\mathrm{Mg}$-doped $\mathrm{GaN}$ samples, only a single exponential decay component with a lifetime $\tau=160 \mathrm{ps}$ is observed (Fig. 1). As explained earlier, ${ }^{11}$ heavily $\mathrm{Mg}$-doped $\mathrm{GaN}$ is free of $\mathrm{Ga}$ vacancies trapping positrons, and the lifetime $\tau$ $=\tau_{B}=160 \mathrm{ps}$ corresponds to positrons annihilating as delocalized particles in the defect-free $\mathrm{GaN}$ lattice. Our result

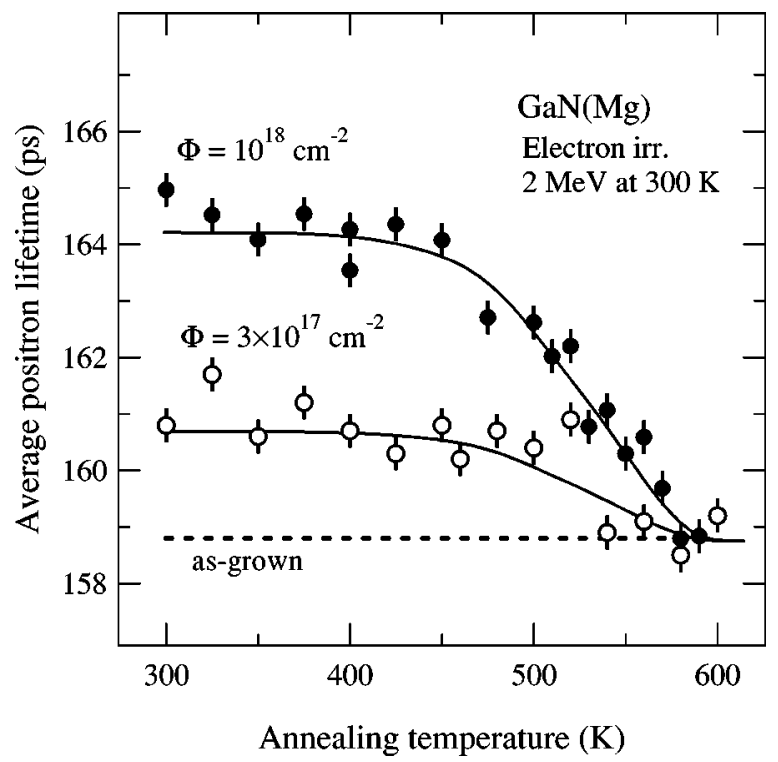

FIG. 2. Average positron lifetime as a function of annealing temperature $T_{\text {ann }}$ in two GaN samples irradiated to fluences $\Phi$. The measurement temperature was $T_{\text {meas }}=300 \mathrm{~K}$. The dashed line shows the level of the average positron lifetime in as-grown samples before irradiation.

$\tau_{B}=160 \mathrm{ps}$ is slightly smaller than that reported previously $(165 \mathrm{ps}) .{ }^{11}$ By measuring several reference samples (e.g., bulk $\mathrm{Si}, \mathrm{GaAs}$, and $\mathrm{InP}$ ), we confirmed that the shift is systematic and constant. We could attribute this to slight differences in the positron lifetime spectrometers, such as scintillators, geometry, and electronic settings. The reproducibility of the positron lifetime experiment, however, is within the statistical error of $\Delta \tau_{\mathrm{av}}=0.3 \mathrm{ps}$.

The 2-MeV electron irradiations, with fluences of $\Phi=3$ $\times 10^{17}$ and $1 \times 10^{18} \mathrm{~cm}^{-2}$, increase the average positron lifetimes by 2 and $5 \mathrm{ps}$, respectively. This increase is a direct evidence of vacancy defects introduced in the samples in the electron irradiation. Figure 2 shows the average positron lifetime $\tau_{\mathrm{av}}$ at $T_{\text {meas }}=300 \mathrm{~K}$ as a function of the annealing temperature $T_{\text {ann }}$. Almost no recovery of $\tau_{\mathrm{av}}$ is observed up to $T_{\text {ann }}=450 \mathrm{~K}$. At $500-600 \mathrm{~K}$ the average lifetime decreases, and finally coincides with the positron lifetime of about $\tau_{B}$ $=160 \mathrm{ps}$ in defect-free GaN. The annealing curve is similar for both irradiations, except that the absolute level of $\tau_{\mathrm{av}}$ is higher for higher fluences. The data of Fig. 2 indicate the recovery of the irradiation-induced vacancy defect at 500$600 \mathrm{~K}$.

In heavily Mg-doped GaN bulk crystals, positrons are efficiently captured by the hydrogenic states around negative Mg ions. ${ }^{11}$ This trapping prevents positrons from getting into the vacancy defects, thus decreasing the average lifetime $\tau_{\mathrm{av}}$. The influence of negative ions on the positron lifetime spectrum becomes stronger at low measurement temperatures, because fewer positrons are able to escape thermally from the ions [binding energy is $\sim 60 \mathrm{meV}$ (Ref. 11)]. The presence of negative $\mathrm{Mg}$ ions was verified in irradiated $\mathrm{GaN}$ crystals of this work by observing a strong decrease of $\tau_{\mathrm{av}}$ when measurement temperature was lowered toward $T_{\text {meas }}$ $=80 \mathrm{~K}$. 
To minimize positron annihilation at $\mathrm{Mg}$ ions and maximize it at vacancies, we performed a high statistics (30 $\times 10^{6}$ counts) positron lifetime experiment at an elevated temperature of $T_{\text {meas }}=400 \mathrm{~K}$ after annealing the sample at $450 \mathrm{~K}$. The lifetime spectrum (Fig. 1) clearly has two exponential components. The shorter component is $\tau_{1}=150$ $\pm 2 \mathrm{ps}$, with an intensity $I_{1}=82 \pm 2 \%$. This is a superposition resulting from positron annihilations either as trapped at negative $\mathrm{Mg}_{\mathrm{Ga}}$ ions (the lifetime is $\tau_{\text {ion }}=\tau_{B}=160 \mathrm{ps},{ }^{11}$ as expected for negative ions with no open volume ${ }^{12,13}$ ) or as delocalized particles in the lattice (the lifetime is less than $\tau_{B}=160 \mathrm{ps}$ due to both annihilation and trapping processes). The longer lifetime component $\tau_{2}=235 \pm 5 \mathrm{ps}$, with an intensity $I_{2}=18 \pm 2 \%$, corresponds to positrons trapped at the irradiation-induced vacancy defect.

According to our earlier studies, ${ }^{3,11}$ the positron lifetime spectrum in $n$-type GaN bulk crystals contains two components, the longer of which is associated with positrons trapped at native vacancy defects. As demonstrated with similar decay slopes in Fig. 1, the positron lifetime at native vacancies in $n$-type $\mathrm{GaN}$ is the same $\left(\tau_{2}=235 \pm 5 \mathrm{ps}\right)$ as that found in this work after electron irradiation. This means that the native vacancy is the same defect as that introduced by electron irradiation at $300 \mathrm{~K}$, i.e., the open volume in both of them corresponds to a single missing atom from the lattice site.

The open volumes at $\mathrm{Ga}$ and $\mathrm{N}$ vacancies are very different because $\mathrm{N}$ is a much smaller atom than $\mathrm{Ga}$. This difference is directly reflected in the positron lifetimes. According to theoretical calculations ${ }^{14}$ the unrelaxed $\mathrm{N}$ and Ga vacancies yield positron lifetimes of 160 and 209 ps, respectively. Furthermore, the calculated lifetime for the Ga vacancy ${ }^{14}$ becomes equal to the experimental value of $235 \pm 5$ ps if we take into account that the $\mathrm{N}$ atoms surrounding the $\mathrm{Ga}$ vacancy relax outward by $5-10 \%$ of the bond length. ${ }^{1,2}$ The experimental positron lifetime of $\tau_{V}=\tau_{2}=235 \pm 5 \mathrm{ps}$ can thus be attributed to the Ga vacancy, but not with the $\mathrm{N}$ vacancy. ${ }^{14}$ The same identification can be unambiguously made with the Doppler broadening spectroscopy. ${ }^{3,14}$ Further, the nitrogen vacancies are expected to be positively charged and thus repulsive to positrons in highly resistive GaN. ${ }^{1,2} \mathrm{Ga}$ vacancies are thus observed in positron lifetime experiments both in $n$-type $\mathrm{GaN}$ bulk crystals as well as in Mg-doped crystals after $2-\mathrm{MeV}$ electron irradiation at $300 \mathrm{~K}$.

The results of Fig. 2 demonstrate that the concentration of $\mathrm{Ga}$ vacancies in $2-\mathrm{MeV}$ electron irradiation increases with the fluence. The quantitative analysis of the $V_{\mathrm{Ga}}$ concentrations and the introduction rate can be done with the positron trapping model. ${ }^{12}$ When positrons annihilate in the lattice (lifetime $\tau_{B}$ ), at negative ions $\left(\tau_{\text {ion }}=\tau_{B}\right.$ ), and at Ga vacancies $\left(\tau_{V}\right)$ the average positron lifetime can be written simply as $\tau_{\mathrm{av}}=\left(1-\eta_{V}\right) \tau_{B}+\eta_{V} \tau_{V}$, where $\eta_{V}$ is the fraction of positron annihilations at vacancies: ${ }^{12}$

$$
\eta_{V}=\frac{\mu_{V} c_{V}}{\tau_{B}^{-1}+\mu_{V} c_{V}+\mu_{\text {ion }} c_{\text {ion }} /\left(1+\delta \tau_{B}\right)} .
$$

We use the positron trapping coefficients $\mu_{V}=\mu_{\text {ion }}=3$ $\times 10^{15} \mathrm{~s}^{-1}$ at $300 \mathrm{~K}$ (Ref. 11) for Ga vacancies and negative

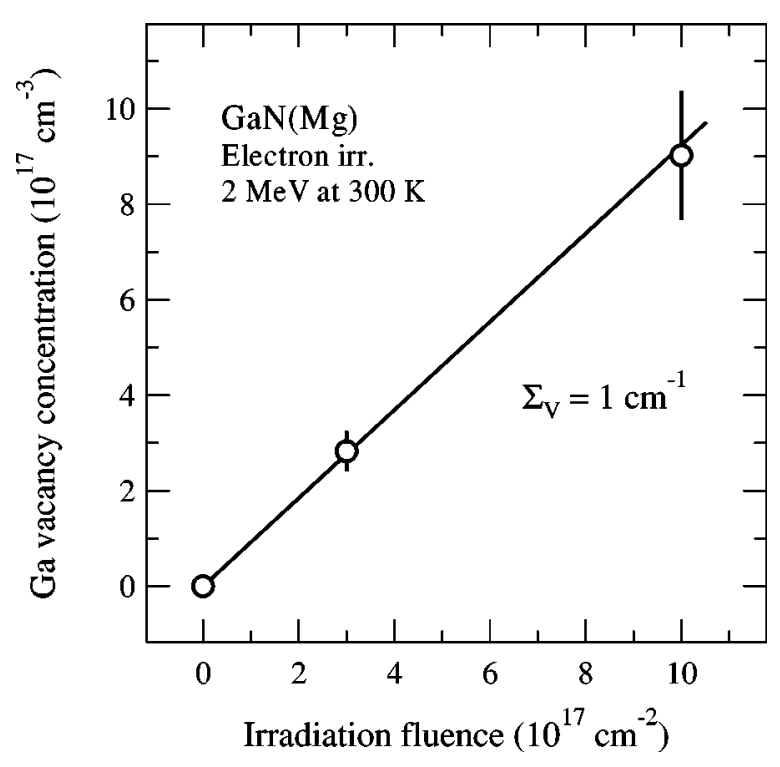

FIG. 3. The concentration of Ga vacancies as a function of a $2-\mathrm{MeV}$ electron irradiation fluence at $300 \mathrm{~K}$. The solid line corresponds to the introduction rate of $\Sigma_{V}=1 \mathrm{~cm}^{-1}$.

$\mathrm{Mg}_{\mathrm{Ga}}$ ions. The positron detrapping rate from the ions can be expressed as $\delta(T) \propto \mu_{\text {ion }} T^{-3 / 2} \exp \left(-E_{\text {ion }} / k_{B} T\right)$, where $E_{\text {ion }}$ $=60 \mathrm{meV}$ (Ref. 11) is the positron binding energy at the Rydberg state of the $\mathrm{Mg}_{\mathrm{Ga}}$ ions. When the concentration of negative ions is fixed to the $\mathrm{Mg}$ concentration $c_{\text {ion }}$ $=10^{20} \mathrm{~cm}^{-3}$ determined in these crystals using secondaryion mass spectrometry, ${ }^{11}$ we can solve the Ga vacancy concentrations from the average positron lifetime and Eq. (1). Note that the possible irradiation-induced negative ions do not play any role in this analysis, because the concentration of negative $\mathrm{Mg}_{\mathrm{Ga}}$ in the samples is $10-100$ times larger than that of any defect formed in the irradiation.

Figure 3 shows that the concentration of $\mathrm{Ga}$ vacancies increases linearly with the electron irradiation fluence in the range $\Phi=(0-10) \times 10^{17} \mathrm{~cm}^{-2}$. The introduction rate is roughly $\Sigma_{V}=c_{V} / \Phi=1 \mathrm{~cm}^{-1}$. This is a typical value for primary defects formed in the irradiation. Furthermore, the same introduction rate has been determined for Frenkel pairs in the nitrogen sublattice using $0.7-1-\mathrm{MeV}$ irradiation. ${ }^{5}$ For $2-\mathrm{MeV}$ electrons the electrical experiments show a total acceptor introduction rate of $1-2 \mathrm{~cm}^{-1} \cdot{ }^{15}$ This value is close to that determined here for Ga vacancies, but other acceptors, such as the N interstitials, ${ }^{5}$ most likely also contribute to the total introduction rate of acceptors.

Chow et al. ${ }^{6}$ recently applied optical detection of electron paramagnetic resonance to observe the interstitial $\mathrm{Ga}$ and related defects in $\mathrm{GaN}$ after $2.5-\mathrm{MeV}$ electron irradiation. According to their results, the Ga interstitial is stable with annealing up to room temperature, where it becomes mobile and subsequently trapped, perhaps by oxygen impurities in the sample. It is clear that this trapped Ga interstitial must have left a Ga vacancy behind in the irradiation. Furthermore, the high concentration of oxygen [about $10^{20} \mathrm{~cm}^{-3}$ (Ref. 11)] enables an efficient trapping of Ga interstitials in our samples. In perfect agreement with the results of Chow 
et al., ${ }^{6}$ we thus detect an irradiation-induced Ga vacancy after a similar $2-\mathrm{MeV}$ irradiation at $300 \mathrm{~K}$.

The results of Fig. 2 indicate that Ga vacancies recover in 30 -min isochronal heat treatments at 500-600 K. At this temperature, the electrical experiments of Look et al. ${ }^{5}$ showed an increase in electron mobility, most likely due to the recovery of irradiation-induced defects acting as carrier scattering centers. The defects associated with trapped $\mathrm{Ga}$ interstitials are stable at $500-600 \mathrm{~K}$, and anneal at clearly higher temperatures of 700-900 K according to the ODEPR experiments of Bozdog et al..$^{7}$ and Linde et al. ${ }^{8}$ This suggests that $\mathrm{Ga}$ vacancies recover by becoming mobile defects at 500-600 K. Assuming that the vacancy takes roughly $10^{3}-10^{5}$ jumps with an attempt frequency $10^{13} \mathrm{~s}^{-1}$ in the 30 -min annealing time, we can convert the recovery temperature of $600 \mathrm{~K}$ to an activation energy of 1.5 (2) eV. This value can be taken as an estimate of the migration energy of Ga vacancies in $\mathrm{GaN}$.

The native $\mathrm{Ga}$ vacancies observed in as-grown $n$-type $\mathrm{GaN}$ are stable up to much higher temperatures than $600 \mathrm{~K}$, where the irradiation-induced Ga vacancies recover (Fig. 2). This points out directly that the Ga vacancies formed during the growth are not isolated but belong to stable defect complexes, most likely with $\mathrm{O}$ impurities as suggested earlier. ${ }^{16}$ At growth temperatures (typically $>1300 \mathrm{~K}$ ), negatively charged $\mathrm{Ga}$ vacancies are mobile and migrate in the lattice until they are trapped at positive oxygen donors. In fact, our preliminary results show that native Ga vacancy complexes recover in heat treatments at $1300-1500 \mathrm{~K}$ in samples grown by metal-organic chemical vapor deposition at about $1300 \mathrm{~K}$.
The recovery temperature of $1400 \mathrm{~K}$ corresponds to an activation energy of 3.7 (2) $\mathrm{eV}$, which can be interpreted as the sum $E_{M}+E_{B}$ of the migration energy of $V_{\mathrm{Ga}}$ and the binding energy of the $V_{\mathrm{Ga}}-\mathrm{O}_{\mathrm{N}}$ complex. Taking the migration energy $E_{M}=1.5(2) \mathrm{eV}$ determined above, we obtain a binding energy of $E_{B}=2.2(4) \mathrm{eV}$ for the $V_{\mathrm{Ga}}-\mathrm{O}_{\mathrm{N}}$ pair. This value is in excellent agreement with the theoretical estimates of $1.8-2.1 \mathrm{eV}^{1,2}$

In summary, we have obtained direct evidence of the formation and thermal stability of $\mathrm{Ga}$ vacancies in $2-\mathrm{MeV}$ electron-irradiated GaN bulk crystals. The introduction rate of Ga vacancies is about $1 \mathrm{~cm}^{-1}$, which is a typical value for primary defects, and close to those obtained previously for $\mathrm{N}$ vacancies and interstitials in $0.7-1 \mathrm{MeV}$ irradiations. ${ }^{5}$ The $\mathrm{Ga}$ vacancies recover in heat treatments at $500-600 \mathrm{~K}$. According to previous ODEPR experiments, ${ }^{6-8} \mathrm{Ga}$ interstitials are trapped by other defects at this temperature range and anneal only above $700 \mathrm{~K}$. We thus conclude that Ga vacancies become mobile defects at 500-600 K, and recover in long-range migration processes, with an estimated migration energy of 1.5 (2) eV. These results indicate that the native $\mathrm{Ga}$ vacancies formed during the crystal growth are associated with defect complexes, probably with the oxygen donors. We estimate a binding energy of 2.2 (4) eV for these complexes, indicating that they are stable at the elevated growth temperature of $\mathrm{GaN}$.

D. C. L. was supported under U.S. Air Force Contract No F33615-00-C-5402.
${ }^{1}$ J. Neugebauer and C. Van de Walle, Appl. Phys. Lett. 69, 503 (1996).

${ }^{2}$ T. Mattila and R. M. Nieminen, Phys. Rev. B 55, 9571 (1997).

${ }^{3}$ K. Saarinen, T. Laine, S. Kuisma, J. Nissilä, P. Hautojärvi, L. Dobrzynski, J. M. Baranowski, K. Pakula, R. Stepniewski, M. Wojdak, A. Wysmolek, T. Suski, M. Leszczynski, I. Grzegory, and S. Porowski, Phys. Rev. Lett. 79, 3030 (1997).

${ }^{4}$ K. Saarinen, in III-Nitride Semiconductors: Electrical, Structural and Defects Properties, edited by M. O. Manasreh (Elsevier, Amsterdam, 2000), p. 109.

${ }^{5}$ D. C. Look, D. C. Reynolds, J. W. Hemsky, J. R. Sizelove, R. L. Jones, and R. J. Molnar, Phys. Rev. Lett. 79, 2273 (1997).

${ }^{6}$ K. H. Chow, G. D. Watkins, A. Usui, and M. Mizuta, Phys. Rev. Lett. 85, 2761 (2000).

${ }^{7}$ C. Bozdog, H. Przybylinska, G. D. Watkins, V. Härle, F. Scholz, M. Mayer, M. Kamp, R. J. Molnar, A. E. Wickenden, D. D. Koleske, and R. L. Henry, Phys. Rev. B 59, 12479 (1999).

${ }^{8}$ M. Linde, S. J. Uftring, G. D. Watkins, V. Härle, and F. Scholz, Phys. Rev. B 55, R10 177 (1997).

${ }^{9}$ W. M. Chen, I. A. Buyanova, M. Wagner, B. Monemar, J. L.
Lindström, H. Amano, and I. Akasaki, Phys. Rev. B 58, R13 351 (1998).

${ }^{10} \mathrm{~S}$. Porowski and I. Grzegory, in GaN and Related Materials, edited by S. J. Pearton (Gordon and Breach, Amsterdam, 1997), Vol. 2, p. 295.

${ }^{11}$ K. Saarinen, J. Nissilä, P. Hautojärvi, J. Likonen, T. Suski, I. Grzegory, B. Lucznik, and S. Porowski, Appl. Phys. Lett. 75, 2441 (1999).

${ }^{12}$ K. Saarinen, P. Hautojärvi, and C. Corbel, in Identification of Defects in Semiconductors, edited by M. Stavola (Academic, New York, 1998), p. 209.

${ }^{13}$ R. Krause-Rehberg and H. S. Leipner, Positron Annihilation in Semiconductors (Springer, Heidelberg, 1999).

${ }^{14}$ K. Saarinen, J. Nissilä, J. Oila, V. Ranki, M. Hakala, M. J. Puska, P. Hautojärvi, J. Likonen, T. Suski, I. Grzegory, B. Lucznik, and S. Porowski, Physica B 273-274, 33 (1999).

${ }^{15}$ D. C. Look (unpublished).

${ }^{16}$ J. Oila, V. Ranki, K. Saarinen, P. Hautojärvi, J. Likonen, J. M. Baranowski, K. Pakula, M. Leszczynski, and I. Grzegory, Phys. Rev. B 63, 045205 (2001). 